\title{
Études post-AMM : apport dans la réinscription des produits
}

\author{
Mathieu Molimard ${ }^{1}$, Marion Bamberger ${ }^{2}$ et Muriel Vray ${ }^{3}$ et les participants à la table ronde $n^{\circ} 6$ de Giens XXIV ${ }^{\star}$ \\ 1 Département de Pharmacologie, CHU de Bordeaux, Bordeaux, France \\ 2 Laboratoire Bristol Myers Squibb, Rueil-Malmaison, France \\ 3 Unité d'Épidémiologie des Maladies Émergentes, Institut Pasteur, Paris, France
}

Texte reçu le 15 octobre 2008 ; accepté le 20 novembre 2008

\begin{abstract}
Mots clés :
pharmaco

épidémiologie ;

remboursement ;

renouvellement

d'inscription

Résumé - Les études post-inscription documentent, en situation réelle, les conditions de mise sous traitement, les populations réellement traitées, les durées de traitement, l'observance, les bénéfices des traitements, l'impact du traitement sur les stratégies thérapeutiques, l'organisation des soins et l'impact de santé publique, ... Leurs données sont utilisées notamment lors d'une réévaluation anticipée ou lors du renouvellement d'inscription au remboursement. Cent trente quatre demandes de la Commission de la Transparence (CT) et/ou du Comité Économique des Produits de Santé (CEPS) ont été faites depuis 1997 et leurs résultats sont pris en compte et participent à la réévaluation du Service Médical Rendu (SMR) et de l'Amélioration du Service Médical Rendu (ASMR) anticipés. Au cours de cette table ronde des Rencontres Nationales de Pharmacologie Clinique de Giens, les difficultés de réalisation des études post-inscription médicaments ont été identifiées et des propositions ont été formulées dans les domaines du dialogue autour de la demande, de la prévisibilité et de l'anticipation des demandes et de la formation des médecins.
\end{abstract}

\section{Introduction}

La mesure des effets des médicaments doit se poursuivre tout au long de leur vie. Elle débute en préclinique par la mesure des effets pharmacologiques, se poursuit jusqu'à la première inscription sur la liste des médicaments remboursables aux assurés sociaux pour mesurer l'effet clinique et le rapport bénéfice risque puis dans les études post-inscription pour fournir au cours des réinscriptions successives les mesures de l'efficacité en vie réelle. Le but des études post-inscription est de documenter, en situation réelle, les conditions de mise sous traitement, les populations traitées, les durées de traitement, l'observance, les bénéfices des traitements, l'impact du traitement sur les stratégies thérapeutiques, l'organisation des soins et l'impact de santé publique, ... Les données sont utilisées notamment lors d'une réévaluation anticipée ou lors du renouvellement.

\footnotetext{
* Pour la liste des participants, voir en fin d'article.
}

\section{Bilan de la demande}

À l'occasion de leur inscription sur la liste des médicaments remboursables, certains médicaments font l'objet d'une demande d'étude post-inscription.

En effet, lors de l'évaluation par la Commission de la transparence (CT), les données disponibles, obtenues dans des essais cliniques, sont parfois limitées. Par définition, ces essais ont eu lieu dans des conditions et sur des populations de patients particuliers. Il est alors nécessaire de compléter ces informations ou d'étudier leur transposabilité en pratique réelle. Ces informations apportées par ces études permettront d'éclairer la Commission dans sa réévaluation, du niveau du SMR, d'ASMR et des conditions de prises en charge du médicament.

Ces études sont demandées principalement par la Commission de la transparence mais peuvent l'être également par le Ministère de la santé [Direction Générale de la Santé (DGS) et Direction de la sécurité sociale (DSS)] et le Comité Économique des 
ÉTUDES POST-INSCRIPTION (CT et/ou CEPS)

134 demandes effectives en août 2008 (depuis 1997)

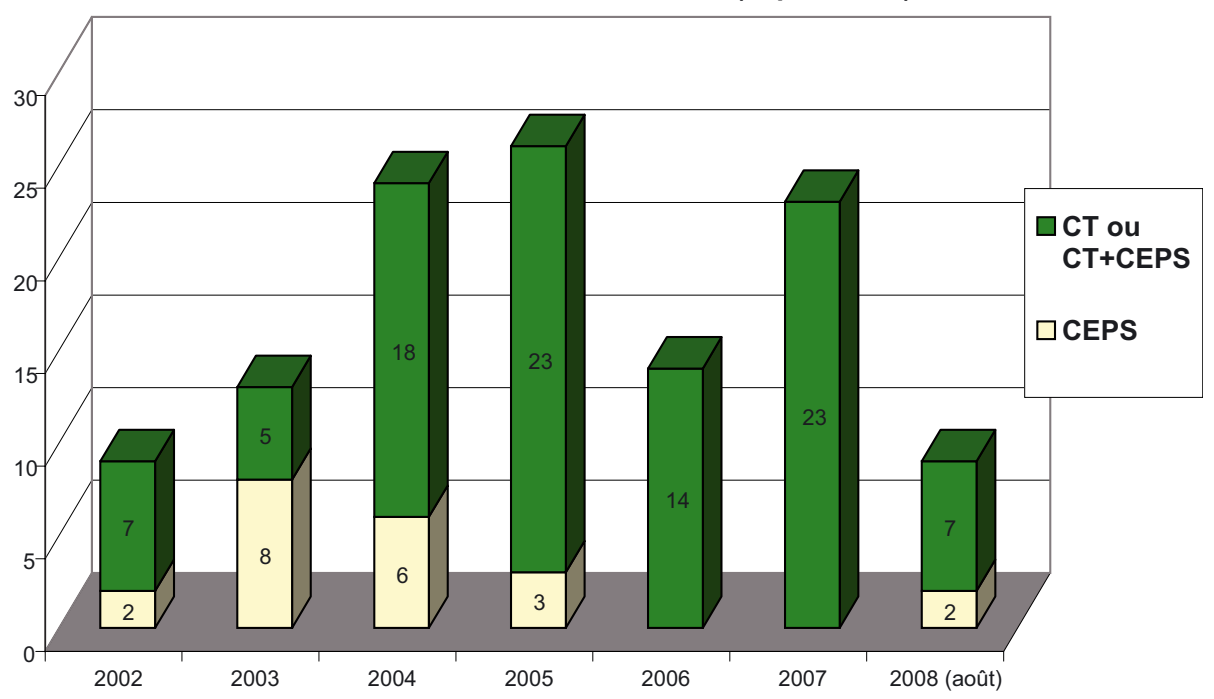

CT : Commission de la Transparence ; CEPS : Comité Économique des Produits de Santé

Fig. 1. Nombre de demandes effectives d'études post-inscription à fin août 2008.

Produits de Santé (CEPS). Les demandes d'étude et leurs objectifs sont inscrits dans l'avis rendu par la CT et repris dans la convention financière signée entre l'industriel et le CEPS lors de la fixation du prix du produit dans le respect de l'accord cadre signé entre le CEPS et le Leem (Les entreprises du médicament).

Afin de pouvoir mutualiser et harmoniser les demandes, il a été mis en place un comité de liaison réunissant les différents partenaires concernés par ces études [DGS, DSS, HAS (Haute Autorité de Santé), Afssaps (Agence française de sécurité sanitaire des produits de santé), CEPS, INVS (Institut National de Veille Sanitaire), CNAMTS (Caisse Nationale d'Assurance Maladie des Travailleurs Salariés)... ]. Parallèlement, il existe un comité de coordination technique entre la HAS et l'Afssaps afin d'étudier l'articulation entre l'étude post-inscription demandée et une éventuelle étude issue d'un PGR (Plan de Gestion du Risque) européen ou de l'Afssaps.

Le suivi des demandes d'étude et de leur mise en place est effectué par la HAS, qui joue le rôle de «guichet unique». Il revient à l'industriel de réaliser l'étude post-inscription demandée. Il doit dans un premier temps constituer un comité scientifique, responsable notamment de la rédaction du protocole et du déroulement de l'étude. La composition du comité scientifique et le protocole envisagé sont déposés, pour validation, auprès de la CT. La Commission, par l'intermédiaire de son groupe de travail «ISP » (Intérêt de Santé Publique), va donner un avis sur la composition du comité scientifique et sur l'adéquation du protocole proposé à la demande d'étude initiale. Plusieurs versions du protocole sont parfois nécessaires pour obtenir un avis favorable.

Par la suite, l'étude est mise en place par l'industriel. Sa durée est variable, de plusieurs mois à plusieurs années. Une fois l'étude terminée, les résultats sont déposés à la CT, sous forme d'un rapport qui est analysé par le Groupe ISP. Une synthèse des résultats est portée à la connaissance de la Commission, qui l'intégrera à son avis et en tirera les conclusions nécessaires.

De 1997 (date des premières demandes) à fin août 2008, on recense 134 demandes effectives d'études provenant de la CT et/ou du CEPS (figure 1). Ces demandes concernent 105 spécialités différentes commercialisées par 49 laboratoires. Une spécialité est concernée par une seule demande d'étude dans $85 \%$ des cas. Quand il existe plusieurs demandes par spécialité, elles concernent le plus souvent des indications différentes. Les laboratoires n'ont eu qu'une ou deux études à réaliser dans $63 \%$ des cas et $18 \%$ ont eu plus de 4 études à mettre en place.

Ces demandes concernent dans $59 \%$ des cas des produits avec une ASMR I, II et III et des produits sans ASMR (niveau V) dans $23 \%$ des cas. Les produits concernés ont un SMR important dans $85 \%$ des cas.

Ces demandes d'études concernent des spécialités issues de nombreuses classes thérapeutiques différentes, mais quatre d'entre elles représentent près des deux tiers (63\%) des demandes : antinéoplasiques, anti-infectieux, médicaments du système nerveux central et médicaments hormonaux (hors hormones sexuelles). Pour cinq classes thérapeutiques ou de médicaments 


\title{
ÉTUDES POST-INSCRIPTION (CT et/ou CEPS) 134 demandes effectives en août 2008 (depuis 1997)
}

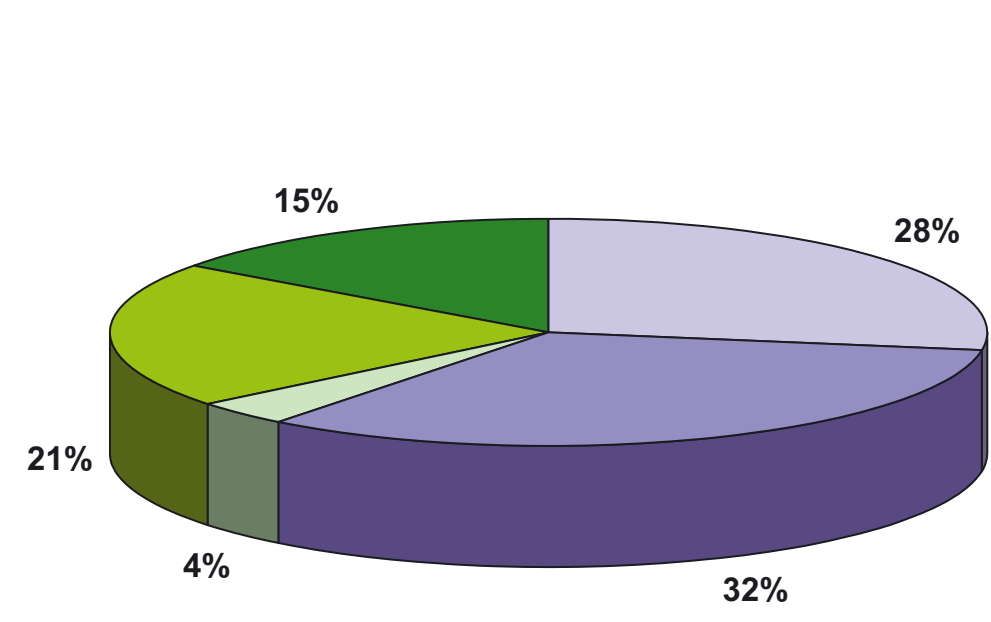

\author{
$\square$ Études terminées \\ ou Résultats déposés \\ Études en cours de \\ réalisation \\ $\square$ Protocoles validés ou \\ Examen final du \\ protocole effectué \\ $\square$ Protocoles en cours de \\ validation
}

Aucun document reçu

Fig. 2. État des demandes effectives d'études Post-inscription à fin août 2008.

à même visée thérapeutique, l'ensemble des spécialités ont fait l'objet d'une demande d'étude post-inscription : interférons $\beta$, hormones de croissance hypophysaires, biothérapies en rhumatologie et en dermatologie et médicaments de la dégénérescence maculaire liée à l'age (DMLA).

Les paramètres à étudier peuvent êtres répartis en quatre grandes catégories : les conditions de prescription et d'utilisation (97\% des demandes), le bénéfice pour le patient (80\% des demandes), la tolérance en situation clinique courante et son impact sur le traitement (58\% des demandes) et l'impact du traitement sur le système de soins ( $21 \%$ des demandes). Les études concernent le plus souvent plusieurs paramètres. Celles explorant les conditions d'utilisation et le bénéfice représentent $77 \%$ des cas et celles concernant les quatre catégories $12 \%$ des cas.

À fin août 2008, sur 134 demandes d'études, aucun protocole n'avait été déposé pour 20 d'entres elles (15\%) dont 8 étaient des demandes récentes $(<10$ mois $)$.

Parallèlement, 110 demandes $(85 \%)$ avaient fait l'objet du dépôt d'un protocole, parmi lesquelles : 28 (21\%) avaient leur protocole en cours de validation, 5 (4\%) avaient eu une validation du protocole (l'étude allait débuter), 44 (32\%) avaient leur étude en cours de réalisation, et pour 37 (28\%) des résultats intermédiaires ou définitifs avaient été déposés (dont $57 \%$ de résultats définitifs) (figure 2).

Les impacts de ces études sont nombreux et peuvent être résumés dans le tableau I.

\section{Difficultés rencontrées et solutions proposées}

Plusieurs difficultés pour la réalisation de ces études ont été identifiées et des solutions ont été proposées au cours de la table ronde :

\subsection{Des difficultés autour de la demande}

Parfois, il existe des difficultés concernant la compréhension de la demande ou la formulation de l'objectif. Cette non compréhension peut conduire à proposer des études inadaptées et justifie donc de mettre en place une procédure de clarification et d'accompagnement de la demande. Afin d'améliorer la compréhension de la demande il est proposé d' adjoindre à l'avis de la CT une annexe motivant la demande d'étude. Cette annexe permettra également de pouvoir communiquer le besoin auprès des maisons-mères. Par ailleurs, une fois l'avis de la CT validé, la possibilité de prendre un contact précoce avec le groupe ISP, afin d'obtenir une clarification de l'objectif, devrait être prévu.

L'existence de demandes similaires émanant de différents états membres européens peut également poser des difficultés dans le choix d'une méthodologie d'étude commune. Les demandes nationales multiples font déjà l'objet d'une coordination entre les différents demandeurs dans le cadre du comité de liaison et de coordination. Une structure de coordination des demandes issues des états membres serait utile. 
Tableau I. Impacts des études.

\begin{tabular}{|c|c|}
\hline Institution concernée & Marqueur impacté \\
\hline Afssaps/EMEA & $\begin{array}{l}\text { AMM et bénéfice/risque } \\
\text { Pharmacovigilance / Plan de gestion de risque } \\
\text { Utilisation et bon usage, conditions de prescription et de délivrance } \\
\text { Recommandations }\end{array}$ \\
\hline $\begin{array}{l}\text { HAS : } \\
- \text { CT } \\
\text { - CEESP }\end{array}$ & $\begin{array}{l}\text { SMR } \\
\text { ASMR } \\
\text { Population cible } \\
\text { Intérêt de santé publique } \\
\text { Utilisation et bon usage / fiches debon usage } \\
\text { Recommandations } \\
\text { Évaluation médicoéconomique }\end{array}$ \\
\hline CEPS/Ministre/DGS & $\begin{array}{l}\text { Remboursement } \\
\text { Prix (accords prix volume et autres modalités) } \\
\text { Intérêt de santé publique }\end{array}$ \\
\hline Hôpitaux & Choix des médicaments par les comités des médicaments \\
\hline $\begin{array}{l}\text { Assurances médicales obligatoires } \\
\text { Agences régionales de santé }\end{array}$ & $\begin{array}{l}\text { Efficience } \\
\text { Accords de bon usage } \\
\text { Contrats individuels } \\
\text { Contrats individuels de médecins } \\
\text { Contrats d'objectifs et de bon usage }\end{array}$ \\
\hline
\end{tabular}

Afssaps : Agence française de sécurité sanitaire des produits de santé; EMEA : European Medicines Agency; HAS : Haute Autorité de Santé; CEESP : Commission d'Évaluation Économique et de Santé Publique; CEPS : Comité Économique des Produits de Santé ; DGS : Direction Générale de la Santé; AMM : Autorisation de Mise sur le Marché; SMR : Service Médical Rendu; ASMR : Amélioration du Service Médical Rendu.

\subsection{Des difficultés de financement}

Le coût de ces études doit être anticipée auprès des maisons mères. Ceci passe par une meilleure compréhension du besoin.

La compréhension du besoin et l'anticipation de la demande financière par les maisons-mères pourrait être améliorée par l'annexe motivant l'étude jointe à l'avis de la Commission de transparence et par une amélioration de la prévisibilité des études, les études étant le plus souvent demandées pour des produits : (i) réellement innovants; (ii) avec une population cible large; (iii) évalués sur un critère intermédiaire; (iv) avec un risque de mésusage [AMM (Autorisation de Mise sur le Marché), remboursement]; (v) avec un problème de transposabilité (populations cibles, conditions d'utilisation, observance, ....).

Par ailleurs, la publication du compte-rendu de cette table ronde en anglais dans Thérapie sera un élément de communication des besoins des filiales françaises dans ce domaine auprès des maisons mères.

En ce qui concerne les demandes conjointes auprès de plusieurs industriels, il est à noter que ce qui pourrait apparaître comme une simplification est souvent à l'origine de délais supplémentaires nécessaires pour mettre d'accord l'ensemble des partenaires.

Enfin, le coût des études peut être élevé au regard du chiffre d'affaire attendu et nécessite dans tous les cas une anticipation du financement. En cas de disproportion du budget nécessaire à la réalisation de l'étude par rapport au chiffre d'affaire attendu, il existe déjà la possibilité de déduction en crédit de remise qui peut être discuté dans la convention avec le CEPS. Le financement sera amélioré par l'anticipation par les laboratoires. La mise en place d'accès à des bases de données permettrait également de réduire les coûts mais peu de bases sont accessibles. L'accès aux bases de l'assurance maladie devrait être possible pour les industriels et la création d'une base de données sur le modèle de la base de donnée de médecine générale anglaise (GPRD - General Pratice Research Database) pourrait être très utile.

\subsection{Des difficultés de réalisation et de délais}

Probablement du fait de la relative nouveauté de ces demandes, celles-ci sont rarement anticipées dans le plan de développement du produit, ce qui est à l'origine du délai entre la demande d'étude et le dépôt du premier protocole. Ce délai peut être amélioré par une meilleure communication autour de la demande et par un contact précoce de clarification de l'objectif, par la mise en valeur du temps des ATU (Autorisations Temporaires d'Utilisation).

Le recours au comité de protection des personnes (CPP) dans le cadre d'étude observationnelle a été fortement discuté pour les délais supplémentaires et les difficultés posés, ces comités n'ayant pas de formation à la pharmaco épidémiologie. Le principe de ces 
études étant de mesurer l'utilisation du médicament en vie réelle, la simple nécessité de signature de consentement demandée par de tels comités serait une source majeure de biais dans la validité de l'observation faite. Les situations nécessitant une intervention (analyse biologique, questionnaire de qualité de vie, ...) sont rares.

Les méthodologies de ces études sont relativement peu connues des équipes médicales plus habituées à faire du développement clinique. L'adaptation à ce type d'étude nécessite l'acquisition de nouvelles compétences. Les méthodes doivent être adaptées à la question posée. Il n'existe pas de conseil scientifique préalable permettant d'orienter ou de valider une orientation méthodologique. Il est proposé de mettre en place des ateliers de formation pratique à l'aide d'exemples.

La participation des médecins et des patients à ce type d'étude est difficile notamment par la méconnaissance du besoin et de l'intérêt de ces études. Il est nécessaire de sensibiliser les médecins à la pharmaco épidémiologie lors de la formation médicale initiale et la formation continue, d'impliquer les sociétés savantes dans ces études (sans qu'elles soient obligatoirement les effecteurs) et de communiquer les résultats et l'apport de ces études aux médecins. La description des résultats dans les avis de transparence permet une communication publique des résultats, en particulier auprès des médecins.

\section{Conclusion}

Au terme de cette table ronde, il apparaît que les études postAMM demandées sont réalisées et prises en compte dans la réévaluation des SMR et ASMR par la Commission de la Transparence ainsi que par le CEPS. Elles permettent d'améliorer la connaissance sur l'utilisation des médicaments en en situation clinique courante. Des difficultés ont été identifiées et des propositions ont été formulées dans les domaines du dialogue autour de la demande, de la prévisibilité des demandes, des coûts des études et de la formation des médecins.

\section{Participants}

Marie-Pierre Allicar (GlaxoSmithKline), Véronique Ameye (Novartis), Bernard Avouac (Hôpital Henri Mondor, Créteil), Marie-Noëlle Banzet (Laboratoires Servier), Laurent Becquemont (Hôpital Bicêtre, Le Kremlin Bicêtre), Patrick Blin (Université Victor Segalen Bordeaux 2, Bordeaux), Corinne Duguay (Sanofi Aventis), Francis Fagnani (CEMKA), Danièle Girault (Wyeth Pharmaceuticals), Muriel Haim (MSD Chibret), Françoise Hamers (Haute Autorité de Santé), Olivier Lalaude (MSD Chibret), Véronique Lamarque (Laboratoire Pfizer), Patricia Maillere (IRIS groupe Servier), Jacques Massol (Hôpital Saint Jacques, Besançon), Philippe Maugendre (Haute Autorité de Santé), Arlette Meyer (Direction Générale de la Santé), Thierry MoreauDefarges (Laboratoire Janssen-Cilag), Atul Pathak (Pharmacologie Clinique, Faculté de Médecine, Toulouse), Anne Perillat (Direction Générale de la Santé), Sophie Ravoire (Laboratoire Lilly), Alain Spriet (Consultant), Sophie Tardieu (AP-HM, Marseille), Bernard Teisseire (CEPS, Ministère de la Santé et des Sports, Paris), Monique Weber (CNAMTS), Laura Zanetti (Afssaps).

Correspondance et offprints : Mathieu Molimard, Département de Pharmacologie, CHU Pellegrin Carreire, 33076 Bordeaux Cedex, France.

E-mail : mathieu.molimard@pharmaco.u-bordeaux2.fr 